\title{
The Use of a Metal Stent in the Treatment of Ureteral Stricture in a Patient Who Had Undergone Radical Cystectomy with the Creation of an Ileal Conduit
}

\author{
Taku Mochizuki Takashi Kawahara Genta Iwamoto \\ Shinnosuke Kuroda Masahiro Yao Hiroji Uemura \\ Departments of Urology and Renal Transplantation, Yokohama City University Medical \\ Center, Yokohama, Japan; Department of Urology, Yokohama City University Graduate \\ School of Medicine, Yokohama, Japan
}

\section{Keywords}

Metal stent $\cdot$ Resonance $\cdot$ Ureteral stricture $\cdot$ Ileal conduit $\cdot$ Antegrade approach

\begin{abstract}
Approximately $9.2 \%$ of patients who undergo radical cystectomy are reported to develop ureteral stricture as a result of both benign and malignant conditions. A metallic stent is a continuous, unfenestrated all-metal double-pigtail ureteric stent with no end or side holes. The patient was a 74-year-old man who underwent radial cystectomy with the creation of an ileal conduit due to advanced bladder cancer 7 years previously. Although he had no radiographic recurrence, he developed bilateral hydronephrosis. We herein report the first case of a patient with ureteral stricture after radial cystectomy with the creation of an ileal conduit who was successfully treated by the insertion of a metal stent by a combined antegrade and retrograde approach.


 Oncology}

\section{Introduction}

Approximately $9.2 \%$ of patients who undergo radical cystectomy are reported to develop ureteral stricture as a result of both benign and malignant conditions [1, 2]. In most cases, ureteral stricture is caused by benign conditions; $16.4 \%$ of patients are reported to develop ureteral stricture due to malignant conditions. The condition is usually first treated by the implantation of a single J stent by a retrograde approach; however, the stent is sometimes bent due to anatomic reasons, necessitating nephrostomy. The Resonance ${ }^{\circledR}$ metal stent is a continuous, unfenestrated, all-metal, double-pigtail ureteric stent with no end or side holes $[3,4]$. We herein report the first case of a patient with ureteral stricture after radial cystectomy with the creation of an ileal conduit who was successfully treated by the insertion of a metal stent with a combined antegrade and retrograde approach.

\section{Case Presentation}

The patient was a 74-year-old man who had undergone radical cystectomy with the creation of an ileal conduit due to advanced bladder cancer 7 years previously. Although he had no radiographic recurrence, he developed bilateral hydronephrosis (Fig. 1a). In February 2017, retrograde ureteroscopy was performed from the ileal conduit and no tumor recurrence was observed. His renal function gradually declined as his serum creatinine level increased to 3.0 with bilateral hydronephrosis. We attempted to insert a single J stent but failed due to severe ureteral obstruction. We therefore performed percutaneous nephrostomy and throughed the guidewire to the ileal conduit (Fig. 2a). We extracted the guidewire from the ileal conduit under cystoscopic guidance (Fig. 2b). We then placed a metallic ureteral stent via a retrograde approach under fluoroscopic guidance (Fig. 2c). His renal function gradually improved and we clamped the nephrostomy. CT revealed no hydronephrosis after clamping. We removed the nephrostomy. His renal function was maintained without hydronephrosis (Fig. 1b).

\section{Discussion}

The implementation of a diversion that requires the use of an appliance, such as an ileal conduit or cutaneous ureterostomy, is primarily considered for patients undergoing radical cystectomy who are not candidates for continent diversion procedures [5]. It is reported that $4-7.9 \%$ of such patients develop ureteroileal obstruction $[6,7]$.

Ureteral stenting allows for drainage from the renal pelvis to the ileal conduit and maintains the renal function. When a patient with an ileal conduit develops ureteroileal obstruction, the physician will often attempt to implant a single J stent under fluoroscopic or cryptoscopic guidance. However, in cases of severe ureteral stenosis, the tension of the single J stent does not keep it in place in the upper ureter, which may result in spinning in the ileal conduit. Furthermore, a single J stent should be exchanged monthly. For severe cases, percutaneous nephrostomy might be an option; however, this reduces the daily quality of life.

Recently, a metallic stent was approved and used clinically for the treatment of ureteral obstruction [3]. The metallic stent is tough due to its spiral shape and the hard material from which it is constructed, and can therefore be left in place for a longer period. We previously demonstrated that a polyurethane ureteral stent should be exchanged due to coloring and 
stone formation $[8,9]$. Thus, the use of a metallic stent can expected to reduce the need for unnecessary ureteral exchanges [10].

In patients with an ileal conduit, frequent ureteral stent exchanges should be avoided to limit the use of general anesthesia. The single J stent is considered the gold standard for the treatment of ureteral obstruction in patients with an ileal conduit; however, a metallic ureteral stent might be an option for the treatment of ureteral stricture when such patients display severe stenosis.

In the present case, the metallic stent was placed via a combined antegrade and retrograde approach. If we had used an antegrade approach alone, it would not be possible to detect the loop on the renal pelvic side. On the other hand, if a retrograde approach alone had been used, the stent would likely have been bent due to the anatomical features. Thus, the simultaneous approach contributed to the control of pyelography from nephrostomy and the through-and-through guide wire maintained its tension, which allowed for the easy advancement of the stent to the renal pelvis.

This was the first case in which a metal stent was successfully inserted via a combined antegrade and retrograde approach for the treatment of ureteral stricture in a patient who had undergone radical cystectomy with the creation of an ileal conduit. This technique might be a new option for the placement of metal stents.

\section{Conclusion}

We reported a case of ureteral stricture in a patient who had undergone radical cystectomy with the creation of an ileal conduit, in whom a metal stent was successfully placed via a combined antegrade and retrograde approach.

\section{Statement of Ethics}

Written informed consent was obtained from the patient for participation in the present study and the publication of the data.

Due to ethical restrictions, the raw data underlying this paper are available upon request from the corresponding author.

\section{Disclosure Statement}

The authors declare no conflicts of interest in association with the present study.

\section{Funding Sources}

The present study was supported by a grant from KAKENHI, a grant from the Ministry of Education, Culture, Sports, Science and Technology of Japan (16K20152) and a grant from the 2016-2017 Research Development Fund (No. WJ2810) of Yokohama City University. 


\section{Case Reports in Oncology}

\begin{tabular}{l|l}
\hline Case Rep Oncol 2018;11:159-163 \\
\hline DOI: 10.1159/000487588 & $\begin{array}{l}\text { @ 2018 The Author(s). Published by S. Karger AG, Basel } \\
\text { www.karger.com/cro }\end{array}$ \\
\hline
\end{tabular}

Mochizuki et al: The Use of a Metal Stent in the Treatment of Ureteral Stricture in a Patient Who Had Undergone Radical Cystectomy with the Creation of an Ileal Conduit

\section{Author Contributions}

T.M. and T.K. conceived and designed the experiments, T.M. and T.K. analyzed the data, T.M., T.K., G.I., S.K., M.Y., and H.U. performed the experiments, and T.M. and T.K. wrote the paper.

\section{References}

1 Westerman ME, Parker WP, Viers BR, Rivera ME, Karnes RJ, Frank I et al. Malignant ureteroenteric anastomotic stricture following radical cystectomy with urinary diversion: patterns, risk factors, and outcomes. Urol Oncol. 2016;34(11):485.e1-e6.

2 Mullins JK, Guzzo TJ, Ball MW, Pierorazio PM, Eifler J, Jarrett TW et al. Ureteral stents placed at the time of urinary diversion decreases postoperative morbidity. Urol Int. 2012;88(1):66-70.

3 Asakawa J, Iguchi T, Tamada S, Ninomiya N, Kato M, Yamasaki T et al. Outcomes of indwelling metallic stents for malignant extrinsic ureteral obstruction. Int J Urol. 2017 Nov. https://doi.org/10.1111/iju.13500.

4 Hsu JS, Huang CY, Liu KL, Chow PM. Risk Factors for Primary Failure of Metallic Ureteral Stents: Experience from a Tertiary Center. J Endourol. 2018 Feb;end.2017.0611.

5 Rodríguez AR, Lockhart A, King J, Wiegand L, Carrion R, Ordorica R et al. Cutaneous ureterostomy technique for adults and effects of ureteral stenting: an alternative to the ileal conduit. J Urol. 2011 Nov;186(5):193943.

6 Vandenbroucke F, Van Poppel H, Vandeursen H, Oyen R, Baert L. Surgical versus endoscopic treatment of non-malignant uretero-ileal anastomotic strictures. Br J Urol. 1993 Apr;71(4):408-12.

7 Kawahara T, Ito H, Terao H, Ogawa T, Uemura H, Kubota Y et al. Ureteroscopy-assisted retrograde nephrostomy for percutaneous nephrolithotomy after urinary diversion. Case Rep Nephrol Urol. 2012 Jul;2(2):113-7.

8 Kawahara T, Ito H, Terao H, Yoshida M, Matsuzaki J. Ureteral stent encrustation, incrustation, and coloring: morbidity related to indwelling times. J Endourol. 2012 Feb;26(2):178-82.

9 Kawahara T, Miyamoto H, Ito H, Terao H, Uemura H, Kubota Y et al. Discolored ureteral stents: findings in urinalysis and urine culture. PLoS One. 2015 Apr;10(4):e0122984.

10 Kawahara T. Editorial Comment from Dr Kawahara to Prevention and treatment of symptoms associated with indwelling ureteral stents: a systematic review. Int J Urol. 2017 Apr;24(4):260.
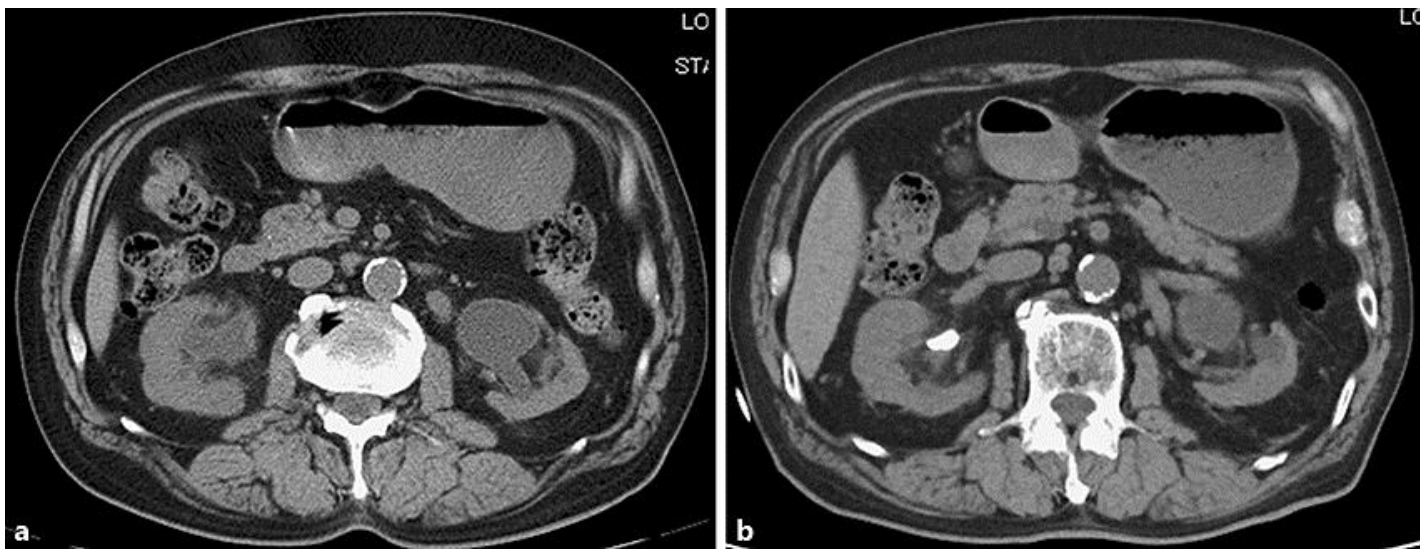

Fig. 1. Pre- (a) and postoperative (b) CT. 


\section{Case Reports in Oncology}
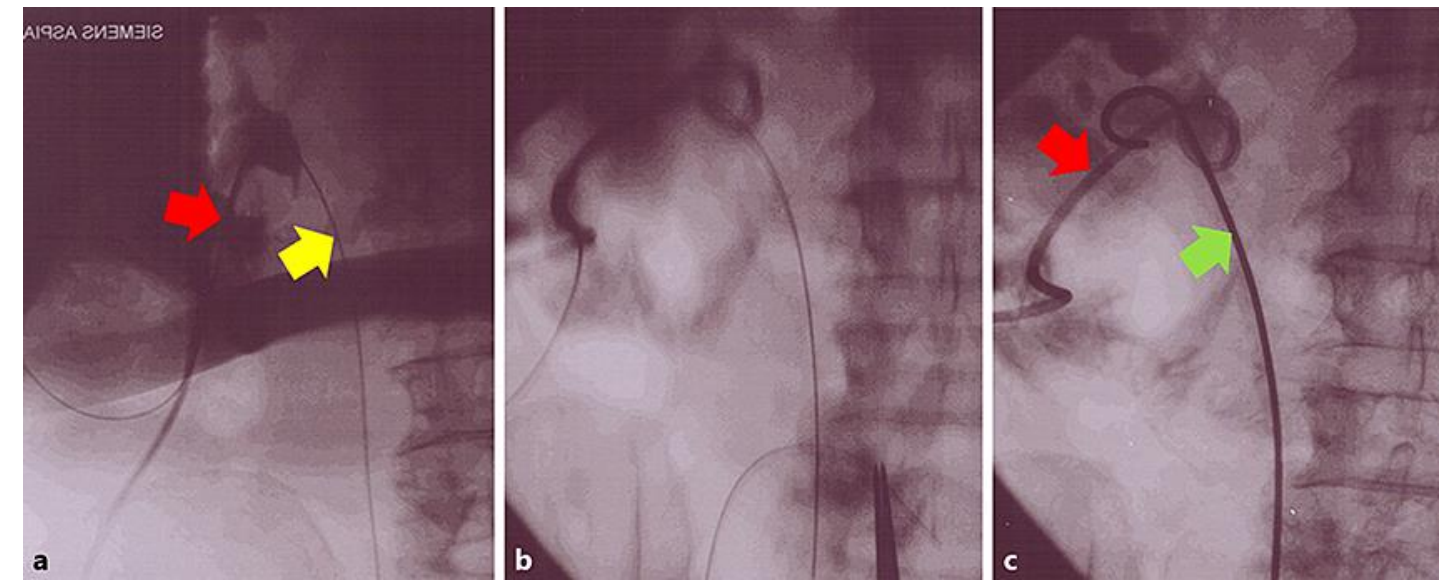

Fig. 2. Making nephrostomy (red arrow) and passing through the guidewire to the ureter (yellow arrow)

(a), grasping the guidewire from ileal conduit (b), and inserting metallic stent via retrograde approach (c). 